\title{
A extração de corrupto, Callichirus major (Say) (Crustacea, Thalassinidea), para uso como isca em praias do litoral do Paraná: as populações exploradas
}

\author{
José R. B. Souza ${ }^{1} \&$ Carlos A. Borzone ${ }^{2}$
}

${ }^{1}$ Departamento de Zoologia, CCB, Universidade Federal de Pernambuco. 50670-920 Recife, Pernambuco, Brasil. Bolsista do CNPq. E-mail: jrbsouza@npd.ufpe.br

${ }^{2}$ Centro de Estudos do Mar, Universidade Federal do Paraná. 83255-000 Pontal do Paraná, Paraná, Brasil. Bolsista do CNPq. E-mail: capborza@ufpr.br

\begin{abstract}
Ghost-shrimp Callichirus major (Say) (Crustacea, Thalassinidea) extraction for bait use in beaches of Paraná's littoral: the exploited populations. A recreational fishery that use ghost-shrimp as bait was evaluated along $11 \mathrm{~km}$ of a sandy beach of Paraná State. The beach was divided in five sectors and samples were obtained along a transect extended on the middle of each sector, after and before months of major extractive activity. The highest densities were found in beaches with well select very fine sands and gentle slope. The density decreased only at the sector that presented greater extractive activity. In this sector, the smallest mean modal size was detected too. The total standing stock of the whole area was $2,486,250$ individuals before summer's months, with a mean density of de 4.48 ind. ${ }^{-2}$. Annual fishing effort represented nearly $10 \%$ of total standing stock.
\end{abstract}

KEY WORDS. Bayt, Crustacea, recreational fishery, sandy beaches, Thalassinidea.

Os crustáceos decápodes cavadores da infraordem Thalassinidea, conhecidos vulgarmente com o nome de "corruptos", vêm sendo há mais de vinte anos capturados e utilizados como isca ao longo das praias oceânicas do litoral brasileiro, desde a costa nordeste até a costa sul. Esta atividade, praticada por pescadores amadores, ficou muito popularizada a partir do uso de uma bomba de sucção manual de construção artesanal que permite a extração dos organismos das suas galerias, além da divulgação de matérias correlatas em revistas de pesca amadora e pela televisão.

Até o momento, foram citadas 42 espécies de Thalassinidea para a costa brasileira (MєLo 1999). Dentre estas, Callichirus major (Say, 1818) é a predominante em praias oceânicas da costa paranaense (Souza \& Borzone 1996). Outros crustáceos deste grupo também são utilizados como isca em outros lugares do mundo. Há registros de intensa exploração de Callianassa australiensis (Dana, 1852) na Austrália (Hailstone \& STEPHENSON 1961), de Callianassa californiensis (Dana, 1854) e Upogebia pugettensis (Dana, 1852) nos Estados Unidos (Dumbauld et al. 1989), e de Callichirus kraussi (Stebbing, 1900) e Upogebia africana (Ortmann) na África do Sul (WYNBERg \& BRANCH 1991).

A pesca destes organismos cavadores pode ocasionar alterações, tanto na espécie alvo, como em outras espécies existentes no sedimento, devido à própria técnica de captura utilizada. $\mathrm{Na}$ África do Sul, a cada 50 corruptos coletados com bomba de sucção, $50 \mathrm{~g}$ de organismos da macroinfauna acabam morrendo ou sendo predados por aves e outros organismos, em conseqüência da perturbação (Wynkerg \& Branch 1991). Por outro lado, um esforço de pesca excessivo pode levar à sobre-exploração do recurso e até ao total desaparecimento das populações alvo. A estrutura populacional de Callichirus major pode ter sido afetada pela pesca no litoral do Estado de São Paulo, onde foi registrada uma diminuição na moda média do tamanho dos indivíduos da população ao longo de 6 anos de estudo. Este resultado pode ser conseqüência de um aumento na intensidade dos recrutamentos, devido à maior disponibilidade de substrato resultante da remoção de indivíduos maiores (Rodrigues \& SHImizu 1997).

Com o objetivo de avaliar a extração de "corruptos" e de conhecer o seu impacto nas populações desse crustáceo nas praias ao sul da desembocadura da Baía de Paranaguá, as mesmas foram divididas em setores em função das condições de acesso dos pescadores, e diversos estudos foram iniciados no ano de 1994. Estudos prévios analisaram a característica da pesca e do pescador ao longo de um ciclo anual, reconhecendo as épocas do ano e os setores de maior extração (Borzone \& SouzA 1996). A dinâmica populacional e a produção secundária desta espécie num setor com baixa intensidade de extração também foram objetos de estudo (Souza et al. 1998). O presente trabalho descreve as características da estrutura populacional de C. major em cada setor acima mencionado, bem como as variações das densidades populacionais antes e depois do período de extração anual de maior intensidade. 


\section{MATERIAL E MÉTODOS}

O trabalho foi desenvolvido ao longo de uma faixa de 11 km de praia, na planície de Praia de Leste ao sul da desembocadura da Baía de Paranaguá. Esta faixa foi dividida em cinco setores: o setor 1, com uma extensão de $1.000 \mathrm{~m}$, representa o limite norte de distribuição da espécie na planície; o setor 2 apresenta $2.100 \mathrm{~m}$ de extensão e encontra-se na frente do bairro conhecido como Asenodi; o setor 3, com 2.400 m de extensão, inclui o Balneário Atami e o seu condomínio de acesso particular e restrito; o setor 4 inclui o Balneário Barrancos, onde existe uma comunidade pesqueira que comercializa os corruptos, e possui $3.100 \mathrm{~m}$ de extensão; finalmente, o setor 5 abrange nos seus $2.600 \mathrm{~m}$ o Balneário de Shangri-lá (Fig. 1). Na região mediana de cada setor foi estabelecida uma transversal perpendicular à praia cobrindo toda a largura do intermareal e com estações de coleta a cada $15 \mathrm{~m}$. Em cada transversal foi levantado um perfil topográfico com teodolito e régua metrada, e coletada uma amostra de sedimento na estação inferior do mediolitoral com um cilindro de $5 \mathrm{~cm}$ de diâmetro e $10 \mathrm{~cm}$ de comprimento. A densidade de corruptos foi estimada através da contagem dos orifícios existentes em $1 \mathrm{~m}^{2}$, com 30 réplicas em cada estação (cada orifício corresponde a um indivíduo de acordo com S. de A. Rodrigues, dados não publicados). A densidade linear foi calculada pela interpolação dos dados de densidade média das estações de cada perfil. Para uma estimativa da abundância total da espécie em cada setor de praia e em cada mês de amostragem, multiplicou-se a densidade linear acima descrita, pelo comprimento em metros de cada setor.

Considerando que a época de extração mais intensa corresponde aos meses de dezembro, janeiro e fevereiro (BORzONE \& Souza 1996), esta amostragem foi realizada no mês de novembro de 1994, e repetida em março e em setembro de 1995. As diferenças de densidade entre os setores e os meses de amostragem foram testadas por análise de variância. Foi utilizado como teste a posteriori o teste de Scheffé (SNEDECOR \& Cochran 1967).

Para conhecer e comparar a estrutura de comprimento em cada setor, na amostragem do mês de setembro de 1995 foi feita uma coleta de indivíduos e utilizado o comprimento da linha talassínica (CLT) como medida para comparação das distribuições de freqüência. Relações morfométricas entre o comprimento da linha talassínica e o comprimento total podem ser consultadas em Souza et al. (1998). O método de Bhattacharya (PAULY \& CADDY 1985) foi utilizado para identificar e determinar os parâmetros estatísticos das diferentes modas apresentadas nos histogramas.

\section{RESULTADOS}

A inclinação do perfil mediolitoral pode ser considerada como suave em todos os setores, existindo um aumento da mesma do setor 1 ao setor 5 (Tab. I). O tamanho do grão do sedimento (média f) aumentou do setor 1 (areia muito fina) ao 5 (areia fina), junto com uma diminuição do grau de seleção (desvio f) do setor 2 ao 5. A largura da região intermareal esteve sempre acima dos 100 metros, mas mostrando uma diminuição do setor 1 ao 5 (Tab. I). As variações de perfil topográfico entre os meses de amostragem mostrou ser diferente entre os setores de praia. O setor 1 apresentou as variações mais drásticas, com uma forte tendência à acrescer após os meses de verão. O setor 3 teve pouca variação após o verão, mas mos-

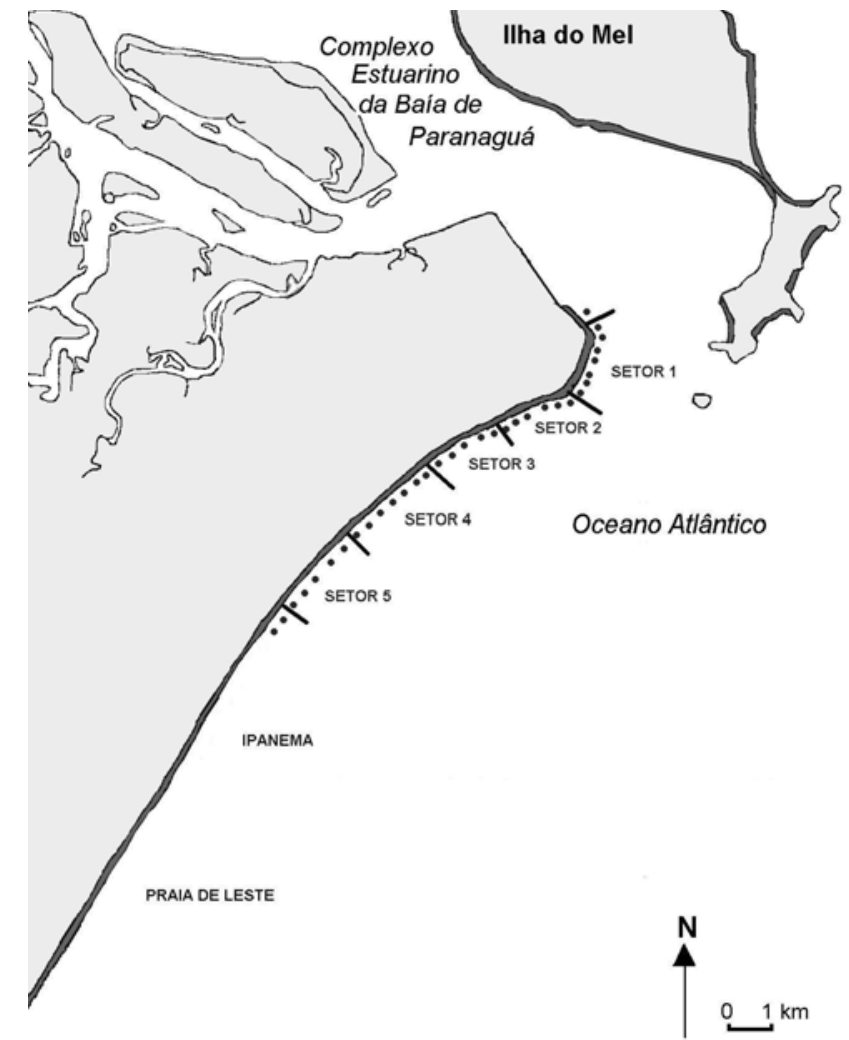

Figura 1. Área de estudo e setores de coleta.

Tabela I. Características gerais do sedimento (região inferior do mediolitoral) e do perfil praial (inclinação e largura do intermareal) nos cinco setores estudados (coleta de novembro de 1994).

$\operatorname{Md}(\phi)=$ diâmetro médio do grão em escala "phi", s $(\phi)=$ desvio em escala $(\phi)$.

\begin{tabular}{ccccc}
\hline Setor & Md $(\phi)$ & $S(\phi)$ & Inclinação $\left(^{\circ}\right)$ & Largura $(\mathrm{m})$ \\
\hline 1 & 2,84 & 0,44 & 0,66 & 160 \\
2 & 3,10 & 0,31 & 0,96 & 115 \\
3 & 2.80 & 0,48 & 1,03 & 130 \\
4 & 2,72 & 0,57 & 1,41 & 105 \\
5 & 1,96 & 0,66 & 1,23 & 105 \\
\hline
\end{tabular}

trou um acréscimo importante no perfil de setembro. Já os setores 2 , 4 e 5 tiveram algumas variações em locais diferentes do perfil, todas após o inverno (Fig. 2).

As maiores densidades médias de $C$. major foram encontradas nos setores $2\left(9,3\right.$ orifícios. $\left.\mathrm{m}^{-2}\right)$ e $3\left(8,5\right.$ orifícios. $\left.\mathrm{m}^{-2}\right)$ e as menores no setor $1\left(0,6\right.$ orifícios. $\left.\mathrm{m}^{-2}\right)$ (Tab. II). A análise de variância multifatorial dos valores de densidade de corrupto mostrou a existência de diferenças significativas entre meses de amostragem e setores (Tab. III). As densidades foram signifi- 

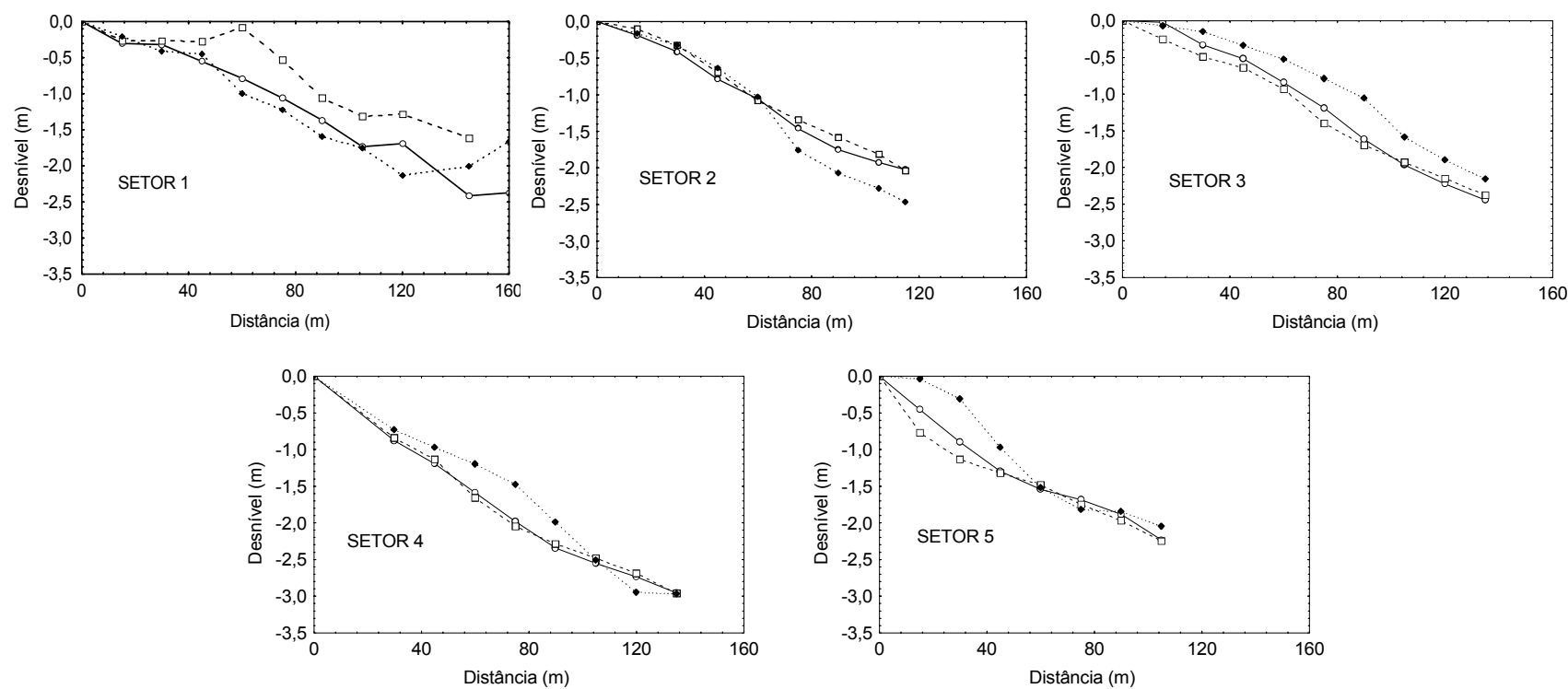

Figura 2. Perfis topográficos de novembro de $1994($ (O), março de $1995(\square)$ e setembro de 1995 ( $)$ ), em cada um dos cinco setores amostrados.

Tabela II. Densidade média $\left(\delta=\right.$ indivíduos. $\left.\mathrm{m}^{-2}\right)$, desvio padrão (D.P.), número de amostras $(\mathrm{N})$ e largura da faixa ocupada por Callichirus major (L), em metros, nos setores estudados.

\begin{tabular}{|c|c|c|c|c|c|c|c|c|c|c|c|c|c|c|}
\hline \multirow[t]{2}{*}{ Setores } & \multicolumn{4}{|c|}{ Novembro/1994 } & \multicolumn{4}{|c|}{ Março/1995 } & \multicolumn{4}{|c|}{ Setembro/1995 } & \multicolumn{2}{|c|}{ Total } \\
\hline & $\delta$ & D.P. & $\mathrm{N}$ & $\mathrm{L}$ & $\delta$ & D.P. & $\mathrm{N}$ & $\mathrm{L}$ & $\delta$ & D.P. & $\mathrm{N}$ & L & $\delta$ & D.P. \\
\hline 1 & 0,9 & 0,9 & 120 & 75 & 0,3 & 0,6 & 90 & 45 & 0,3 & 0,5 & 120 & 60 & 0,6 & 0,80 \\
\hline 2 & 11,1 & 5,8 & 90 & 45 & 8,4 & 5,7 & 120 & 60 & 8,9 & 3,7 & 120 & 60 & 9,3 & 5,20 \\
\hline 3 & 6,2 & 4,8 & 90 & 45 & 11,2 & 4,1 & 120 & 60 & 8,0 & 3,0 & 120 & 75 & 8,5 & 4,60 \\
\hline 4 & 1,5 & 1,4 & 120 & 60 & 1,9 & 1,8 & 120 & 60 & 3,5 & 2,7 & 90 & 45 & 2,2 & 2,13 \\
\hline 5 & 2,7 & 2,3 & 90 & 60 & 4,9 & 2,7 & 90 & 60 & 4,8 & 3,6 & 120 & 60 & 4,1 & 3,10 \\
\hline
\end{tabular}

Tabela III. Resultados da análise de variância multifatorial (MANOVA) da abundância de Callichirus major, considerando os três meses de amostragem e os cinco setores estudados como fatores de variação. (QM) Quadrados médios, (GL) graus de liberdade.

\begin{tabular}{lrrrr}
\hline $\begin{array}{c}\text { Fatores de } \\
\text { Variação }\end{array}$ & GL & \multicolumn{1}{c}{ QM } & \multicolumn{1}{c}{$F$} & $\mathrm{~F}>\mathrm{F}$ \\
\hline Mês (1) & 2 & 105,69 & 9,65 & 0,001 \\
Setor (2) & 4 & 4814,39 & 439,60 & 0,001 \\
1 vs 2 & 8 & 269,00 & 24,56 & 0,001 \\
\hline Resíduos & 1635 & 10,95 & & \\
\hline
\end{tabular}

cativamente maiores no final do verão, não ocorrendo diferença significativa entre final do verão e primavera (Fig. 3). A densidade foi significativamente diferente entre os setores, com exceção dos setores 2 e 3 (Fig. 4). Quando analisadas por separado, podemos ver que as densidades do setor 2 tiveram, ao contrário dos setores 3 , 4 e 5, uma diminuição significativa após o verão (Fig. 5).

O estoque estimado em toda a área estudada a partir do cálculo da densidade linear para cada setor multiplicado pela extensão do setor foi de 2.486.250 indivíduos, com uma densidade média de 4,48 ind. $\mathrm{m}^{-2}$ antes do verão, e de 3.802.500 indivíduos com uma densidade média de 5,34 ind. $\mathrm{m}^{-2}$ no final do verão (Tab. IV). No mês de setembro não houve muita variação a respeito do final do verão, com um total de 3.816 .400 indivíduos e densidade média de 4,94 ind. $\mathrm{m}^{-2}$.

Os histogramas de freqüência de comprimento mostram que existem diferenças entre setores (Fig. 6). Há uma moda principal (segunda moda), com diferenças significativas do valor da sua média entre setores, tendo o menor valor no setor 2 (Tab. V). Uma outra moda (primeira moda) pode ser estatisticamente discriminada apenas nos setores 3 , 4 e 5 . 

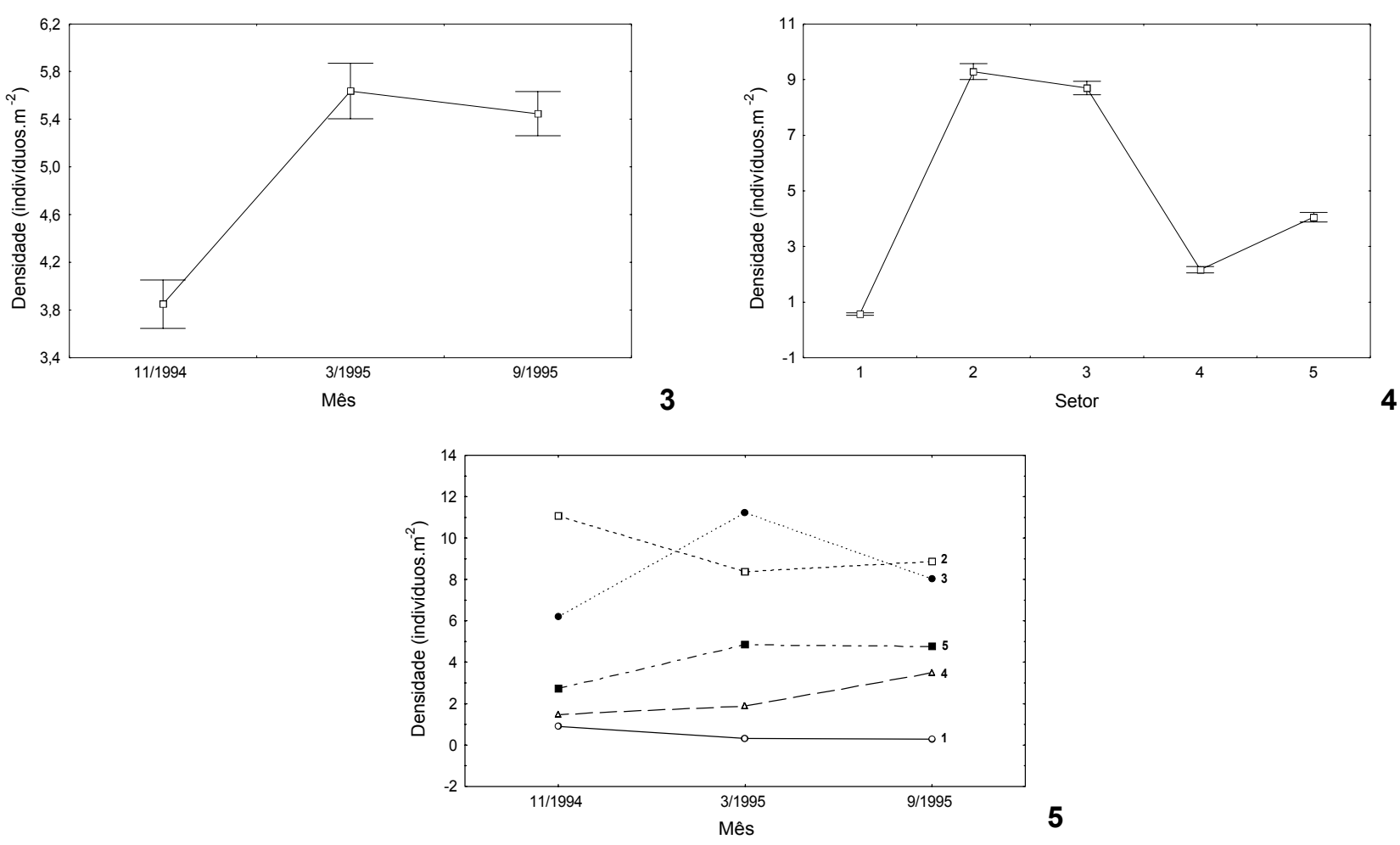

Figuras 3-5. Média e desvio padrão da densidade de Callichirus major por: 3) mês de amostragem, 4) setor estudado e 5) combinação dos dois fatores. Setor $1(O)$, setor $2(\square)$, setor $3(\bullet)$, setor $4(\triangle)$ e setor $5(\square)$.
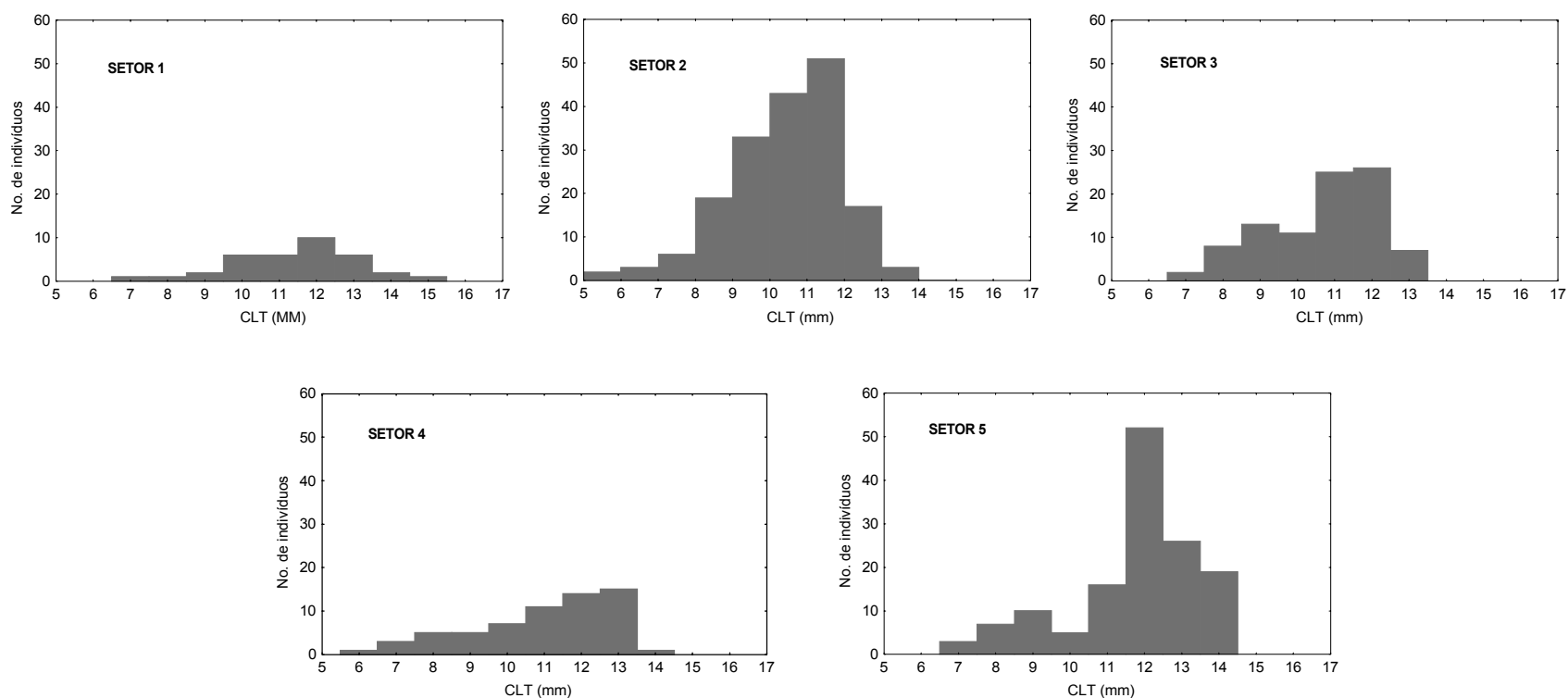

Figura 6. Distribuição de freqüência de comprimento da linha talassínica (CLT) de Callichirus major capturados em cada setor da área de estudo, em setembro de 1995.

Revista Brasileira de Zoologia 20 (4): 625-630, dezembro 2003 


\section{DISCUSSÃO}

As maiores densidades de $C$. major ocorreram em dois setores próximos à desembocadura da Baía de Paranaguá (2 e 3). Estes dois setores apresentam características de praia adequadas para a ocorrência de altas densidades da espécie, com sedimento de areia muito fina e bem selecionada, baixa inclinação e zona de arrebentação de ondas bem definida, como já observado por SouzA \& Borzone (1996). O setor 1, que apresentou as menores densidades, encontra-se no próprio pontal da desembocadura da baía, onde existe uma forte influência das correntes de maré que determinam uma maior dinâmica ambiental. Este setor sofre grandes variações da linha de costa interanualmente (ANGulo \& ARAújo 1996), e os resultados deste trabalho mostram que os perfis da praia podem sofrer fortes variações, principalmente durante os meses de verão, quando ocorre o recrutamento da espécie. Já a diminuição das densidades nos setores 4 e 5 não pode ser explicada por variações sazonais do perfil praial, que foram pequenas, mas pode estar associada a uma mudança nas características físicas da praia, como o aumento do tamanho médio do grau do sedimento junto com a diminuição da seleção, e o aumento da inclinação do perfil praial. Estas características ficam mais pronunciadas ao sul do setor 5, sendo que a espécie praticamente desaparece do intermareal dois quilômetros ao sul deste último ponto estudado.

A diferente intensidade da pesca entre setores não explica as diferenças encontradas nas densidades de corrupto. A pesca foi mais intensa nos setores 2,4 e 5 (Borzone \& SouzA 1996), no entanto o setor 2 apresentou as maiores densidades de corrupto. Por outro lado, este mesmo setor foi o único que mostrou uma diminuição das densidades após o período de extração mais intensa durante o verão, além de ter o menor tamanho médio de corruptos. Podemos inferir que, mesmo sendo o setor com melhores condições naturais para a ocorrência de altas densidades de corruptos, a população deste setor estaria sendo afetada pela extração.

Quanto ao motivo pelo qual o número elevado de pescadores dos setores 4 e 5 não estaria afetando, nem a densidade nem o tamanho médio das populações nestes setores, pode ser explicado por uma deficiência na estimação do esforço de pesca feito no trabalho de Borzone \& Souza (1996). Nesse trabalho foi apenas contabilizado o número de pescadores, mas não a quantidade exata de corrupto extraído por cada pescador. Observações de campo indicam que nestes setores os pescadores podem realizar capturas menores, já que eles permanecem no setor pescando e coletando à medida que vão pescando. Desta forma, eles capturariam bem menos corruptos do que os pescadores que se deslocam para a Baía de Paranaguá após uma grande captura de organismos e que são os usuais freqüentadores do setor 2 .

O estudo das características populacionais de C. major feita no setor 3 (Souza et al. 1998) mostrou que fêmeas ovígeras ocorrem principalmente no mês de dezembro. Isso significa que o recrutamento da espécie na área de estudo estaria ocorrendo no final do verão, o que poderia explicar o aumento nas densidades observado na maioria dos setores estudados.

Borzone \& Souza (1996) estimaram em 5.386 pescadores.ano ${ }^{-1}$ o esforço de pesca do corrupto, com uma CPUE (captura por unidade de esforço) média de 46,61 indivíduos por pescador. Mesmo com este valor de CPUE, que possivelmente esteja superestimado, teríamos uma captura anual total de
251.041 organismos, o que representa aproximadamente uma mortalidade por pesca de $10 \%$ do total de corruptos na área. A partir destes dados podemos concluir que a pesca de corrupto ainda não está afetando drasticamente a população de $C$. major na região estudada, existindo sim certos indícios de algum impacto para o setor 2, como maior incidência de juvenis e menor tamanho médio dos indivíduos. Se esta pescaria mantiver as atuais características, amadora e sazonal, ela provavelmente permanecerá em equilíbrio. É importante ressaltar que, apesar da pesca do corrupto ser recreativa e não gerar renda diretamente através de venda ou produção, ela possui importância econômica já que é um componente significativo das atividades de lazer e de pesca desportiva no litoral, atividades de grande impacto econômico para o Estado de Paraná.

\section{AGRADECIMENTOS}

À Dra. Setuko Masunari pela leitura crítica do manuscrito e sugestões. O presente trabalho foi em parte financiado pelo Conselho Nacional de Desenvolvimento Cientifico e Tecnológico (CNPq) através das bolsas de Doutorado (JRBS) e de Produtividade em Pesquisa (CAB) concedidas.

\section{REFERÊNCIAS BIBLIOGRÁFICAS}

Angulo, R.J. \& A.D. Araujo. 1996. Classificação da costa paranaense com base na sua dinâmica, como subsidio a ocupação da orla litorânea. Boletim Paranaense de Geociências, Curitiba, 44: 7-17.

Borzone, C.A. \& J.R.B. Souza. 1996. A extração de corrupto, Callichirus major (Decapoda: Callianassidae) para uso como isca em praias do litoral do Paraná: Características da pesca. Nerítica, Curitiba, 10: 69-79.

Dumbauld, B.R.; D.A. ARMSTRong \& D.C. Doty. 1989. burrowing shrimp: new bait fishery resource and historical pest to the oyster industry: a preliminary look at their biology in Washington coastal estuaries. Journal of Shelfish Research, Southampton, 8 (1): 320.

Hailstone, T.S. \& W. STEPhenson. 1961. The biology of Callianassa (Trypaea) australiensis Dana, 1852 (Crustacea, Thalassinidea). University of Queensland papers, Brisbane, 1 (12): 259285.

Melo, G.A.S. 1999. Manual de identificação dos Crustacea Decapoda do litoral brasileiro: Anomura, Thalassinidea, Palinuridea e Astacidea. São Paulo, Plêiade/FAPESP, 551p.

Pauly, D. \& J.F. Caddy. 1985. A modification of Bhattacharya's method for the analysis of mixtures of normal distributions. FAO Fisheries Circular \# 781, 16p.

Rodrigues, S.A. \& R.M. Shimizu. 1997. Autoecologia de Callichirus major (Say, 1818), p. 155-170. In: R. ABSALÃo \& A.M. ESTEVES (Eds). Ecologia de praias arenosas do litoral brasileiro. Rio de Janeiro, PPGE-UFRJ, Série Oecologia Brasiliensis, vol. 3, 270p.

Snedecor, G.W. \& W.G. Cochran. 1967. Statistical Methods. Iowa, University Press, 703p.

SouzA, J.R.B. \& C.A. Borzone. 1996. Distribuição de Calianassídeos (Crustacea: Decapoda: Thalassinidea) em praias do litoral paranaense, com especial referência a Callichirus major (Say, 1818). Arquivos de Biologia e Tecnologia, Curitiba, 39 (3): 553-565.

Revista Brasileira de Zoologia 20 (4): 625-630, dezembro 2003 
SouZA, J.R.B.; C.A. Borzone \& T. Brey. 1998. Population dynamics and secondary production of Callichirus major (Crustacea: Thalassinidea) on a southern Brazilian sandy beach. Archives of Fishery and Marine Research, Nehren, 46 (2): 151-164.
WynBerg, R.P. \& G.M. Branch. 1991. An assessment of baitcollecting for Callianassa kraussi Stebbing in Langebaan Lagoon, western Cape, and of associated avian predation. South African Journal of Marine Sciences, Cape Town, 11: 141-152.

Recebido em 30.V.2003; aceito em 20.X.2003.

Revista Brasileira de Zoologia 20 (4): 625-630, dezembro 2003 